\title{
An interacting scenario for dark energy in a Bianchi type-I universe
}

\begin{abstract}
We study the interaction between dark energy (DE) and dark matter in the scope of anisotropic Bianchi type-I space-time. First we derive the general form of the DE equation of state (EoS) parameter in both non-interacting and interacting cases and then we examine its future by applying a hyperbolic scale factor. It is shown that in the non-interacting case, depending on the value of the anisotropy parameter $\mathrm{K}$, the DE EoS parameter varies from phantom to quintessence whereas in the interacting case the EoS parameter varies in the quintessence region. However, in both cases, the DE EoS parameter wde ultimately (i.e. at $\mathrm{z}$ $=-1)$ tends to the cosmological constant $(\omega \mathrm{de}=-1)$. Moreover, we fix the cosmological bound on the anisotropy parameter $\mathrm{K}$ by using recent observational data about the Hubble parameter.
\end{abstract}

Keyword: Cosmology; Bianchi type-I model; Dark energy; Dark matter 